\title{
Merging gauge coupling constants without Grand Unification
}

\author{
F.R. Klinkhamer* \\ Institute for Theoretical Physics, University of Karlsruhe (TH), \\ 76128 Karlsruhe, Germany \\ and \\ G.E. Volovik ${ }^{\dagger}$ \\ Low Temperature Laboratory, Helsinki University of Technology, \\ P.O. Box 2200, FIN-02015 HUT, Finland; \\ L.D. Landau Institute for Theoretical Physics, Russian Academy of Sciences, \\ Kosygina 2, 119334 Moscow, Russia \\ (preprint hep-ph/0505033, KA-TP-06-2005)
}

\begin{abstract}
The merging of the running couplings constants of the weak, strong, and electromagnetic fields does not require the unification of these gauge fields at high energy. It can, in fact, be the property of a general fermionic system in which gauge bosons are not fundamental.
\end{abstract}

PACS 11.10.Hi, 12.10.Kt, 12.60.Re, 67.90.+z, 71.10.-w

\footnotetext{
*Email address: frans.klinkhamer@physik.uni-karlsruhe.de

${ }^{\dagger}$ Email address: volovik@boojum.hut.fi
} 


\section{Introduction}

There are several lessons to be learnt from the example of relativistic quantum fields emerging in condensed matter. One of them is that the physical cutoff can be different for bosons and fermions if the fermions are more fundamental than the bosons. This occurs in superfluid ${ }^{3} \mathrm{He}-\mathrm{A}$, where bosons are the collective modes of the fermionic quantum vacuum and are composite objects made of fermionic degrees of freedom [1]. The naive counting of fermionic and bosonic contributions to the vacuum polarization suggests that the anti-screening effect of charged bosons must dominate over the screening effect of the fermionic vacuum and that, therefore, the effective $S U(2)$ gauge field emerging in ${ }^{3} \mathrm{He}-\mathrm{A}$ must experience asymptotic freedom [2].

However, this is not what happens in superfluid ${ }^{3} \mathrm{He}-\mathrm{A}$. Instead, the $S U(2)$ coupling constant shows the same zero-charge effect as the Abelian $U(1)$ field. ${ }^{1}$ The reason is the difference in cutoff scales for bosons and fermions. As a result, the contribution of the fermions to the logarithmically running coupling constant prevails, in spite of the larger boson content. Actually, the hierarchy of cutoff scales in ${ }^{3} \mathrm{He}-\mathrm{A}$ is such that the asymptotic-freedom contribution from the gauge bosons just does not develop and the only contribution to the vacuum polarization comes from the fermions.

Another important lesson from condensed-matter physics is that the bare coupling constant is absent for emergent gauge fields of a fermionic quantum vacuum. The reason is simply that such gauge bosons cannot exist as free fields, that is, without having fermions around to make the quantum vacuum. This implies, in particular, that the entire gauge coupling constant comes from vacuum polarization.

Here, we assume that the Standard Model of elementary particle physics also has different physical cutoff scales: the compositeness scale $E_{\mathrm{c}}$ which provides the cutoff for the gauge bosons and the much higher ultraviolet cutoff $E_{\mathrm{UV}}$ for the fermions. Assuming that all three coupling constants of the Standard Model come exclusively from vacuum polarization, we will find that the most natural choice for the compositeness scale $E_{\mathrm{c}}$ is the Planck scale $E_{\text {Planck }} \approx 10^{19} \mathrm{GeV}$ (or, possibly, a scale lower by a factor of about $10^{4}$ ), while the ultraviolet cutoff scale $E_{\mathrm{UV}}$ will turn out to be much larger than the Planck scale.

This second cutoff may be associated with the energy scale where Lorentz invariance is violated, $E_{\mathrm{UV}} \sim E_{\mathrm{Lorentz}}$. It has been claimed [3] that cosmic-ray observations imply $E_{\text {Lorentz }}>10^{21} \mathrm{GeV}$, assuming the absence of very small numerical factors in the dis-

\footnotetext{
${ }^{1}$ The term "zero-charge effect" refers to the infrared behavior, whereas "asymptotic freedom" refers to the ultraviolet behavior.
} 
persion relations. ${ }^{2}$ Probably, $E_{\text {Lorentz }}$ is even larger. This would mean that the Planck cutoff is highly Lorentz invariant and that the underlying symmetry of the fundamental structure is itself the Lorentz symmetry, which then protects the Lorentz invariance of the effective low-energy physics [5].

If $E_{\text {Lorentz }} \gg E_{\text {Planck }}$, the topological Fermi-point scenario of emergent relativistic quantum fields may be relevant [1]. Specifically, the integration over fermions with energy $E \lesssim E_{\text {Planck }}$ occurs in the fully relativistic region, where fermions are still close to the Fermi points and, therefore, have gauge invariance and general covariance. As a result, the induced effective action for the gauge and gravity fields is automatically invariant.

The small ratio of cutoff parameters, $E_{\text {Planck }}^{2} / E_{\text {Lorentz }}^{2}$, protects the Lorentz invariance of the known physical laws. This would be in accordance with Bjorken's suggestion [6] that a highly accurate relativistic quantum field theory can only emerge if there is a small expansion parameter in the theory.

The merging of gauge coupling constants at high energy is usually associated with Grand Unification of weak, strong, and electroweak interactions into a larger gauge group with a single coupling constant [7, 8). The two-scale scenario discussed in the present Letter demonstrates that the merging of running couplings could occur without unification, it could very well be the natural property of an underlying fermionic vacuum.

\section{Running couplings from two energy scales}

Let us assume that the gauge fields of the Standard Model are not fundamental but induced, so that the three running coupling constants $g_{i}$ of the gauge group $U(1) \times$ $S U(2) \times S U(3)$ only come from vacuum polarization. In other words, the fine structure constants $\alpha_{i} \equiv g_{i}^{2} / 4 \pi$, for $i=1,2,3$, are completely determined by logarithms and have vanishing bare coupling constants, $1 / \alpha_{i}^{(0)}=0$.

If gauge bosons are fermion composites, the ultraviolet cutoff scale for the vacuum polarization caused by fermions must be larger than the one caused by gauge bosons. Let $E_{\mathrm{UV}}$ be the cutoff for the fermions and $E_{\mathrm{c}} \ll E_{\mathrm{UV}}$ the compositeness scale which provides the cutoff energy for the gauge bosons. Then, for energies above the electroweak

\footnotetext{
${ }^{2} \mathrm{An}$ explicit calculation of photon propagation in a static background of randomly positioned wormholes has shown how, in principle, small numerical factors could appear in the photon dispersion relation 4, but this calculation does not apply to fermions.
} 
scale but below the compositeness scale, one has at one loop (cf. Refs. [8, 9]):

$$
\begin{aligned}
\alpha_{1}^{-1}(E) & =\frac{5 N_{F}}{9 \pi} \ln \frac{E_{\mathrm{UV}}^{2}}{E^{2}} \\
\alpha_{2}^{-1}(E) & =\frac{N_{F}}{3 \pi} \ln \frac{E_{\mathrm{UV}}^{2}}{E^{2}}-\frac{11}{6 \pi} \ln \frac{E_{\mathrm{c}}^{2}}{E^{2}}, \\
\alpha_{3}^{-1}(E) & =\frac{N_{F}}{3 \pi} \ln \frac{E_{\mathrm{UV}}^{2}}{E^{2}}-\frac{11}{4 \pi} \ln \frac{E_{\mathrm{c}}^{2}}{E^{2}},
\end{aligned}
$$

for $M_{Z} \ll E \ll E_{\mathrm{c}} \ll E_{\mathrm{UV}}$ and natural units with $\hbar=c=1$. Here, $N_{F}$ is the number of fermion families contributing to the positive screening (zero-charge) vacuum polarization, whereas the negative anti-screening (asymptotic-freedom) contribution come from the non-Abelian gauge bosons.

At the compositeness scale $E_{\mathrm{c}}$, the weak and strong inverse couplings, as well as the hypercharge inverse coupling with a factor $3 / 5$, approach the same value,

$$
\frac{3}{5} \alpha_{1}^{-1}\left(E_{\mathrm{c}}\right)=\alpha_{2}^{-1}\left(E_{\mathrm{c}}\right)=\alpha_{3}^{-1}\left(E_{\mathrm{c}}\right)=\frac{N_{F}}{3 \pi} \ln \frac{E_{\mathrm{UV}}^{2}}{E_{\mathrm{c}}^{2}}
$$

Above the compositeness scale, the behavior depends on the details of the dynamics. If the gauge bosons break up for $E>E_{\mathrm{c}}$, the story ends here, at least as far as the gauge bosons are concerned. If, on the other hand, the gauge bosons survive but for some reason do not contribute to the vacuum polarization, the couplings run together as

$$
\frac{3}{5} \alpha_{1}^{-1}(E)=\alpha_{2}^{-1}(E)=\alpha_{3}^{-1}(E)=\frac{N_{F}}{3 \pi} \ln \frac{E_{\mathrm{UV}}^{2}}{E^{2}},
$$

for $E_{\mathrm{c}} \ll E \ll E_{\mathrm{UV}}$. As discussed in the Introduction, a similar situation occurs in superfluid ${ }^{3} \mathrm{He}-\mathrm{A}$, with only fermions contributing to the polarization of the vacuum. In this liquid, the running coupling constant of the effective $S U(2)$ field behaves in exactly the same way as the one of the Abelian $U(1)$ field, that is, it experiences the same zerocharge effect. Of course, as the couplings $\alpha_{i}$ from Eq. (3) grow with energy, higher-order contributions need to be added to the logarithm shown (cf. Ref. [9]).

Let us, first, estimate the compositeness scale $E_{\mathrm{c}}$. This can be done in the same way as the standard calculation of the unification scale (cf. Ref. [9]), i.e., only using the bosonic contributions to the running couplings. One then obtains for the compositeness energy scale the same value as usually assumed to hold for Grand Unified Theories (GUTs). ${ }^{3}$

\footnotetext{
${ }^{3}$ The reason is that the right-hand sides of Eqs. (1 $\mathrm{hbc}$ ) can be written solely in terms of $\ln \left(E_{\mathrm{c}}^{2} / E^{2}\right)$ and $\widetilde{\alpha}^{-1} \equiv N_{F} /(3 \pi) \ln \left(E_{\mathrm{UV}}^{2} / E_{c}^{2}\right)$, with $E_{\mathrm{c}}$ and $\widetilde{\alpha}$ taking the role of the unification energy $E_{\mathrm{GUT}}$ and coupling constant $\alpha_{\mathrm{GUT}}$.
} 
Cancelling out the fermionic contributions from the right-hand sides of Eqs. (1abc), one finds two equations at the electroweak scale $M_{Z}$,

$$
\begin{aligned}
\alpha_{2}^{-1}\left(M_{Z}\right)-\alpha_{3}^{-1}\left(M_{Z}\right) & =\frac{11}{12 \pi} \ln \frac{E_{\mathrm{c}}^{2}}{M_{Z}^{2}}, \\
\frac{3}{5} \alpha_{1}^{-1}\left(M_{Z}\right)-\alpha_{2}^{-1}\left(M_{Z}\right) & =\frac{11}{6 \pi} \ln \frac{E_{\mathrm{c}}^{2}}{M_{Z}^{2}} .
\end{aligned}
$$

Extracting the combination $1 / \alpha_{Q} \equiv 1 / \alpha_{1}+1 / \alpha_{2}$ from these equations, one obtains Eq. (21.5.16) of Ref. 9], which expresses the logarithm in terms of the strong coupling constant $\alpha_{3}$ and the fine structure constant $\alpha_{Q}$ at the electroweak scale,

$$
\ln \frac{E_{\mathrm{c}}^{2}}{M_{Z}^{2}}=\frac{2 \pi}{11 \alpha_{Q}\left(M_{Z}\right)}\left(1-\frac{8}{3} \frac{\alpha_{Q}\left(M_{Z}\right)}{\alpha_{3}\left(M_{Z}\right)}\right) .
$$

Taking the numerical values $\alpha_{3}\left(M_{Z}\right) \approx 0.120$ and $\alpha_{Q}\left(M_{Z}\right) \approx 1 / 128$ at $E=M_{Z} \approx$ $91.2 \mathrm{GeV}$ [9], this gives the following estimate:

$$
\ln \left(E_{\mathrm{c}}^{2} / M_{Z}^{2}\right) \approx 60.4
$$

The compositeness scale $E_{\mathrm{c}}$ is about $10^{15} \mathrm{GeV}$, which is relatively close to the Planck energy scale $E_{\text {Planck }} \equiv \sqrt{\hbar c^{5} / G} \approx 1.22 \times 10^{19} \mathrm{GeV}$.

Let us now estimate the ultraviolet cutoff $E_{\mathrm{UV}}$ for the fermions. From Eqs. (1a) and (1b), the fine structure constant $\alpha_{Q}$ at the electroweak scale reads

$$
\alpha_{Q}^{-1}\left(M_{Z}\right)=\frac{8 N_{F}}{9 \pi} \ln \frac{E_{\mathrm{UV}}^{2}}{M_{Z}^{2}}-\frac{11}{6 \pi} \ln \frac{E_{\mathrm{c}}^{2}}{M_{Z}^{2}}
$$

Using Eq. (5) to eliminate the compositeness scale $E_{\mathrm{c}}$, one obtains

$$
\ln \frac{E_{\mathrm{UV}}^{2}}{M_{Z}^{2}}=\frac{3 \pi}{2 N_{F} \alpha_{Q}\left(M_{Z}\right)}\left(1-\frac{2}{3} \frac{\alpha_{Q}\left(M_{Z}\right)}{\alpha_{3}\left(M_{Z}\right)}\right) .
$$

With the numerical values mentioned above, this gives the following estimate:

$$
\ln \left(E_{\mathrm{UV}}^{2} / M_{Z}^{2}\right) \approx 577 / N_{F}
$$

For $N_{F}=3$, one has $\ln \left(E_{\mathrm{UV}}^{2} / M_{Z}^{2}\right) \approx 192$, so that $E_{\mathrm{UV}} \approx 10^{44} \mathrm{GeV} \gg E_{\mathrm{Planck}}$. For $N_{F}=5$, the fermion scale is still larger than the Planck energy by a factor $10^{8}$. The corresponding running coupling constants are shown in Fig. 1].

We realize, of course, that the renormalization-group equations (1abc), with the numerical values (6) and (9) inserted, give a weak mixing angle at $E=M_{Z}$ somewhat below 


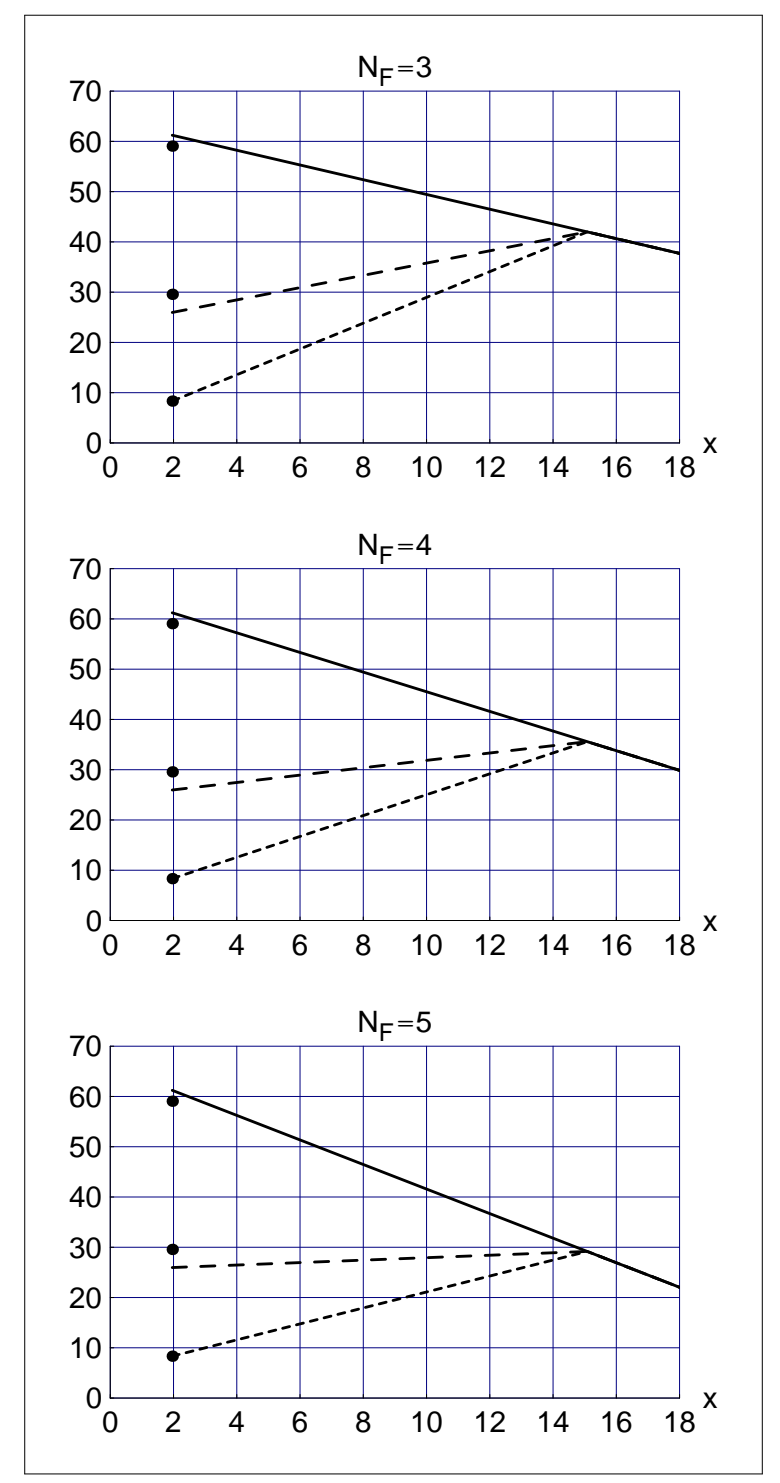

Figure 1: Inverse couplings $(3 / 5) \times \alpha_{1}^{-1}$ [solid curve], $\alpha_{2}^{-1}$ [long-dashed curve], and $\alpha_{3}^{-1}$ [short-dashed curve], as a function of $x \equiv \log _{10}(E / \mathrm{GeV})$ for different numbers $N_{F}$ of fermion families. The coupling constants are given by Eqs. (11), (3), (6), (19), and run together for $E>E_{c}$ [overlapping curves]. At the compositeness scale $E \sim E_{c} \approx 10^{15} \mathrm{GeV}$, there may be threshold effects which somewhat change the values of the couplings towards lower energies (see text). The dots show the experimental values at $E=M_{Z} \approx 91.2 \mathrm{GeV}$. 
the experimental value (cf. Fig. 1). Specifically, we find $\sin ^{2} \theta_{w} \approx 0.203$ instead of the experimental value 0.231 9]. Alternatively, adding appropriate bare coupling constants $1 / \alpha_{i}^{(0)}$ to the right-hand sides of Eqs. (1abc) in order to match the three experimental values at $E=M_{Z}$, we do not find precisely merging coupling constants at high energy.

For a genuine Grand Unified Theory, the problem is serious and has been addressed in different ways; see, e.g., Refs. [10, 11, 12, 13] and references therein. But, for a dynamic scenario as ours, the precise definition of the compositeness scale is rather uncertain. The scale can, in fact, be slightly different for the various composite gauge bosons. In other words, the three couplings of our scenario need not merge exactly at one particular energy (for example, two couplings could merge first and the third one later).

The simplest way to model these threshold effects is to replace $E_{\mathrm{c}}$ in Eqs. (1b) and (1c) by $E_{\mathrm{c} 2}$ and $E_{\mathrm{c} 3}$, respectively, where $E_{\mathrm{c} 2}$ and $E_{\mathrm{c} 3}$ are assumed to be not more than a few orders of magnitude away from the geometric average $E_{\mathrm{c}} \equiv \sqrt{E_{\mathrm{c} 2} E_{\mathrm{c} 3}}$. The experimental values $\alpha_{i}^{\exp }\left(M_{Z}\right)$ then give $\ln \left(E_{\mathrm{c} 2}^{2} / M_{Z}^{2}\right) \approx 50.5, \ln \left(E_{\mathrm{c} 3}^{2} / M_{Z}^{2}\right) \approx 58.0$, and $\ln \left(E_{\mathrm{UV}}^{2} / M_{Z}^{2}\right) \approx$ $557 / N_{F}$. This suggests that the range for threshold effects in $E_{\mathrm{c}}$ may be approximately $10^{13}-10^{15} \mathrm{GeV}$ (which is also clear from Fig. 1 by making appropriate shifts of the curves). Note, that, without grand-unified group, there is no danger of having too rapid proton decay.

\section{Discussion}

Let us end with a few general remarks. Trans-Planckian cutoff scales have been considered before, for example the scale $E_{\text {cutoff }} \approx 10^{42} \mathrm{GeV}$ in Ref. 14] as corresponding to an exotic (non-existing) case. The condensed-matter-like scenario discussed in the present article suggests, however, that this possibility must be taken seriously.

In this scenario, the merging of the running couplings of weak, strong, and hypercharge fields does not require a unification of these fields at high energy, it may simply be the property of a fermionic system in which gauge bosons are not fundamental. The factor $3 / 5$ for $\alpha_{1}^{-1}$ in Eq. (3) may indicate an underlying continuous or discrete symmetry between the fermion species.

The large separation between the Planckian (or near-Planckian) compositeness scale $E_{\mathrm{c}}$ and the trans-Planckian scale $E_{\mathrm{UV}}$ may be of importance to considerations of the Standard Model symmetries as emergent phenomena. In particular, this allows us to discuss gauge invariance as being an emergent symmetry.

In the topological Fermi-point scenario of emergent relativistic fields [1], the spectrum of fermionic excitations near the Fermi point is linear: fermions are chiral and obey the 
relativistic Weyl equation. In this scenario, bosonic excitations behave as effective gauge fields interacting with Weyl fermions. This implies that gauge invariance automatically emerges in the fermionic sector close to the Fermi point, i.e., at $E \ll E_{\mathrm{UV}}$. The fermions induce gauge invariance for the effective action of the composite vector fields. Since the compositeness cutoff parameter $E_{\mathrm{c}}$ is well below $E_{\mathrm{UV}}$, gauge invariance in the bosonic sector is valid throughout the compositeness scale $E_{\mathrm{c}}$. Hence, the requirement suggested by Veltman [15] is fulfilled. Specifically, he concluded that, if gauge bosons are composite, gauge invariance should remain valid both in the infrared $\left(E \ll E_{\mathrm{c}}\right)$ and ultraviolet $\left(E \gg E_{\mathrm{c}}\right)$ regions. The high accuracy of gauge invariance in the bosonic sector is then determined by the small parameter $E_{\mathrm{c}}^{2} / E_{\mathrm{UV}}^{2}$, in accordance with a suggestion of Bjorken 6 .

In the Fermi-point scenario, $E_{\mathrm{UV}}$ is the scale below which the spectrum of fermionic excitations near the Fermi point is linear, i.e., Lorentz invariance induced by the Fermi point is still obeyed. That is why the Lorentz-violation scale must be approximately equal to or larger than $E_{\mathrm{UV}}$. In turn, this implies that Lorentz invariance is more fundamental than the other physical laws and that we cannot expect to observe its violation in the near future.

Applying the two-scale formalism to gravity, one finds that it gives the wrong value for the gravitational coupling constant. If $E_{\mathrm{UV}}$ is again used as the energy cutoff for the fermionic contributions to Newton's constant, one obtains $G^{-1} \sim N_{F} E_{\mathrm{UV}}^{2}$ instead of $G^{-1} \sim N_{F} E_{\text {Planck }}^{2}$. It is not clear at the moment how to cure this problem.

We can only speculate that non-logarithmic (power-law) divergences must be considered with great care. For example, the fourth order divergence, which leads to a vacuum energy density (cosmological constant $\Lambda$ ) of order $E_{\mathrm{UV}}^{4}$ or $E_{\text {Planck }}^{4}$, can be cancelled without fine-tuning, due to the thermodynamic stability of the vacuum [16]. The same may hold for the Higgs mass problem - controlling the quadratically divergent quantum corrections to the Higgs potential mass term (see, e.g., Ref. [17]). This cutoff-dependent mass term is simply absorbed by the vacuum energy density and is zero in the equilibrium vacuum, again due to thermodynamic stability [18]. For induced gravity, the cancellation of the vacuum energy density is demonstrated by a calculation of $\Lambda$ on a $(3+1)$-dimensional brane embedded in $\mathrm{AdS}_{5}$ space: the induced cosmological constant on the brane vanishes without fine-tuning, due to a cancellation of the contributions from $(4+1)$-dimensional fermionic matter and gravity [19].

There may very well be a general principle from the underlying physics, which protects against $E_{\mathrm{UV}}^{n}$ contributions to $G^{-1}$ with $n>0$. Let us mention, in this respect, another example of induced Sakharov gravity in terms of constituent fields, namely Ref. [20], 
which used such a principle and demonstrated the advantage of two energy scales. In the scheme of Ref. [20], the first (lowest) energy scale is the mass scale $M^{\prime}$ of the constituent fields. With $M^{\prime} \sim E_{\text {Planck }}$, this provides a natural cutoff which determines Newton's

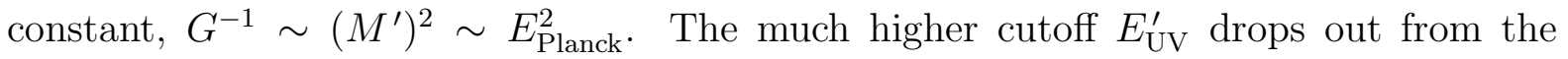
effective action due to imposed cancellations between the constituent fields (see also Ref. [15], where cancellations of fermionic and bosonic effects are required). This scheme only works if Lorentz invariance survives beyond the Planck scale, again in agreement with the statement in Ref. [15] that the symmetry should remain valid throughout the cutoff range. The higher cutoff $E_{\mathrm{UV}}^{\prime}$ of Ref. [20] must, therefore, be below the Lorentz-violation scale.

In conclusion, it is possible that the scenario of emergent physics, in combination with a hierarchy of cutoff energy scales, can replace the grand-unification scenario based on symmetry breaking. This new scenario (with parameters $N_{F}$ and $E_{\mathrm{c}} \ll E_{\mathrm{UV}}$ ) naturally leads to the merging of gauge coupling constants, without the need to introduce a simple gauge group (and without having to worry about too rapid proton decay or too many magnetic monopoles left over from the early universe).

Moreover, the hierarchy of cutoff energy scales may be related to the well-known hierarchy problem of the Standard Model - the absence of a natural explanation for having $M_{Z} \ll E_{\mathrm{GUT}}$ or $E_{\text {Planck }}$. The ${ }^{3} \mathrm{He}-\mathrm{A}$ example mentioned in the Introduction, where gauge invariance is not fundamental, suggests that the mass of the weak vector bosons may result not from spontaneous symmetry breaking but from terms depending on the ultraviolet cutoff. If we accept this viewpoint, the typical value of the weak vector boson mass would be $M_{Z} \sim E_{\mathrm{c}}^{2} / E_{\mathrm{UV}} \ll E_{\mathrm{c}}$, which would be a first step towards understanding the Standard Model hierarchy problem mentioned above (with $E_{\mathrm{c}}$ taking the place of $E_{\mathrm{GUT}}$ ). From the numerical estimates given in Eqs. (6) and (9) and without further threshold effects at the cutoff energies, the suggested hierarchy would seem to prefer having more than $N_{F}=3$ fermion families.

\section{Acknowledgements}

The work of G.E.V. is supported in part by the Russian Ministry of Education and Science, through the Leading Scientific School grant \#2338.2003.2. This work is also supported by the European Science Foundation COSLAB Program. 


\section{References}

[1] G.E. Volovik, The Universe in a Helium Droplet (Clarendon Press, Oxford, 2003).

[2] D.J. Gross and F. Wilczek, "Ultraviolet behavior of non-Abelian gauge theories," Phys. Rev. Lett. 30, 1343 (1973); H.D. Politzer, "Reliable perturbative results for strong interactions?" Phys. Rev. Lett. 30, 1346 (1973).

[3] O. Gagnon and G.D. Moore, "Limits on Lorentz violation from the highest energy cosmic rays?" Phys. Rev. D 70, 065002 (2004), hep-ph/0404196.

[4] F.R. Klinkhamer and C. Rupp, "Spacetime foam, CPT anomaly, and photon propagation," Phys. Rev. D 70, 045020 (2004), hep-th/0312032 F.R. Klinkhamer and C. Rupp, in preparation.

[5] J. Collins, A. Perez, D. Sudarsky, L. Urrutia, and H. Vucetich, "Lorentz invariance: An additional fine-tuning problem," Phys. Rev. Lett. 93, 191301 (2004), gr-qc/0403053 A. Corichi and D. Sudarsky, "New quantum gravity phenomenology," gr-qc/0503078.

[6] J.D. Bjorken, "Emergent gauge bosons," contributed to: 4th Workshop on What Comes Beyond the Standard Model?, Bled, Slovenia, 17-27 July, 2001, hep-th/0111196.

[7] H. Georgi and S.L. Glashow, "Unity of all elementary particle forces," Phys. Rev. Lett. 32, 438 (1974).

[8] H. Georgi, H.R. Quinn, and S. Weinberg, "Hierarchy of interactions in unified gauge theories," Phys. Rev. Lett. 33, 451 (1974).

[9] S. Weinberg, The Quantum Theory of Fields II (Cambridge University Press, Cambridge U.K., 1996).

[10] S. Dimopoulos and H. Georgi, "Softly broken supersymmetry and SU(5)," Nucl. Phys. B 193, 150 (1981); S. Dimopoulos, S. Raby, and F. Wilczek, "Supersymmetry and the scale of unification," Phys. Rev. D 24, 1681 (1981); L.E. Ibanez and G.G. Ross, "Low-energy predictions in supersymmetric grand unified theories," Phys. Lett. B 105, 439 (1981).

[11] U. Amaldi, W. de Boer, and H. Fürstenau, "Comparison of grand unified theories with electroweak and strong coupling constants measured at LEP," Phys. Lett. B 
260, 447 (1991); P. Langacker and N. Polonsky, "Uncertainties in coupling constant unification," Phys. Rev. D 47, 4028 (1993), hep-ph/9210235.

[12] R.N. Mohapatra and M.K. Parida, "Threshold effects on the mass scale predictions in $S O(10)$ models and solar neutrino puzzle," Phys. Rev. D 47, 264 (1993), hep-ph/9204234 D.G. Lee, R.N. Mohapatra, M.K. Parida, and M. Rani, "Predictions for proton lifetime in minimal nonsupersymmetric $S O(10)$ models: An update," Phys. Rev. D 51, 229 (1995), hep-ph/9404238.

[13] Y. Kawamura, "Split multiplets, coupling unification and extra dimension," Prog. Theor. Phys. 105, 691 (2001), hep-ph/0012352

[14] L.V. Laperashvili, H.B. Nielsen, and D A. Ryzhikh, "Monopoles near the Planck scale and unification," Int. J. Mod. Phys. A 18, 4403 (2003), hep-th/0211224 C.R. Das and L.V. Laperashvili, "Phase transition in gauge theories, monopoles and the Multiple Point Principle," hep-ph/0503138.

[15] M. Veltman, "The infrared-ultraviolet connection," Acta Phys. Polon. B12, 437 (1981).

[16] G.E. Volovik, "Cosmological constant and vacuum energy," Ann. Phys. (Leipzig) 14, 165 (2005); gr-qc/0405012.

[17] S. Pokorski, "Phenomenological guide to physics beyond the Standard Model," Lectures given at: NATO Advanced Study Institute and EC Summer School on String Theory: From Gauge Interactions to Cosmology, Cargèse, France, 7-19 June 2004, hep-ph/0502132.

[18] G.E. Volovik, "On contributions of fundamental particles to the vacuum energy," in: I. Ya. Pomeranchuk and Physics at the Turn of the Century, eds. A. Berkov, N. Narozhny, and L. Okun (World Scientific, Singapore, 2003), p. 234, hep-ph/0306011.

[19] A.A. Andrianov, V.A. Andrianov, P. Giacconi, and R. Soldati, "Brane world generation by matter and gravity," hep-th/0503115.

[20] V.P. Frolov, D.V. Fursaev, and A.I. Zelnikov, "Statistical origin of black hole entropy in induced gravity," Nucl. Phys. B 486, 339 (1997), hep-th/9607104. 